\title{
Evaluation of Power System Resilience Improvements in Low-Income Neighborhoods
}

\author{
Laiz Souto \\ Intelligent Systems \& Control Engineering group \\ Universitat de Girona \\ Girona, Spain 17003 \\ laiz.souto@austin.utexas.edu
}

\author{
Surya Santoso \\ Department of Electrical \& Computer Engineering \\ The University of Texas at Austin \\ Austin, Texas 78712-0240 \\ ssantoso@mail.utexas.edu
}

\begin{abstract}
This article provides an evaluation of power system resilience enhancements in low-income neighborhoods. Lowincome households and communities may be subject to risks of different nature, such as natural hazards and human-made attacks, hereby considered as particular cases of high-impact, low-probability events that are highly likely to damage power grid infrastructures. Such events may result in long interruption times and lead to permanent disconnection from the grid in extreme cases, and consequently, the value of load lost may be much higher than the investment cost associated with prevention and mitigation alternatives. In this scenario, this article analyzes the value of load lost and the costs associated with installation, operation, and repair of grid components affected by extreme events to determine the benefits of different strategies for power system resilience improvements targeted at low-income neighborhoods.

Index Terms-power system resilience, cost benefit analysis, extreme weather, microgrids, power distribution, social factors, vandalism
\end{abstract}

\section{INTRODUCTION}

$\mathbf{P}$ OWER grid infrastructures are often vulnerable to a variety of risks that may lead to major disruptions, such as natural hazards, extreme weather conditions, and humanmade attacks. Such occurrences are regarded as high-impact, low-probability events - highly likely to damage power grid infrastructures, albeit unlikely to happen - and may result in long interruption times and huge monetary losses associated with infrastructural damage and the energy not supplied. Thus, enhancing power system resilience is vital to endure these extreme situations and maintain quality and continuity of supply.

Electricity distribution networks in low-income neighborhoods are particularly vulnerable to high-impact, lowprobability risks of different nature - either for inadequate risk assessment for infrastructure planning, or for the impossibility to pay for the repair costs associated with the damages to infrastructure. As a matter of a fact, they usually present a high number of irregular connections, as well as thefts of electricity

This research was supported by the European Union's Horizon 2020 research and innovation programme, call LCE-01-2016-2017, under the auspices of the project "Renewable penetration levered by Efficient Low Voltage Distribution grids", grant agreement number 773715, and University of Girona scholarship. and grid components combined with inefficient energy appliances [1], [2]. As a consequence, they are less reliable than in high-income neighborhoods and may be overloaded at higher ratings than the maximum acceptable limits that ensure a safe operation. To make matters worse, they are often provided with a limited service by the utility company and lack alternatives for energy provision [3].

In the event of an outage in low-income neighborhoods, the supply interruption times may be very long or even permanent due to the impossibility of reconnecting to the grid. As a consequence, the maintenance cost and the value of load lost may be much higher than the investment cost associated with prevention and mitigation alternatives for a resilient grid. On the top of this, the hardships that low-income residents often face due to power outages may be overwhelming, from lack of running water and hot meals for days to destruction of property and loss of life. Therefore, enhancing resilience of low-income neighborhoods is crucial to maintain quality of life and continuity of supply at acceptable levels.

Methodologies for grid resilience improvement under extreme conditions have been presented in [4]-[6], which assessed hardening and operational resilience strategies; [7], which incorporated grid resilience enhancements into planning and operation decisions; in [8], [9], which assessed critical components for improving grid resilience to windstorms; and in [10]-[12] which considered the influence of renewable energy sources for modeling resilience to extreme weather events, among others. However, none of these previous efforts has considered the magnified impacts of an outage caused by high-impact, low-probability events on low-income neighborhoods. Moreover, it is important to highlight that natural hazards, extreme weather conditions, and human-made attacks affect households and external grid components in different ways. Thus, distinct approaches targeted at households and external grid components should be considered to adequately improve power system resilience in low-income neighborhoods.

In this context, this article aims at quantifying the benefits of two strategies for power system resilience improvements targeted at low-income neighborhoods: (1) deploying intelligent electronic devices to detect and eliminate thefts of 
energy (which helps to prevent a particular case of humanmade attacks), and (2) installing off-grid photovoltaic systems or independent generators at household or community level for autonomous operation from the external grid against supply interruption (caused by natural disasters and extreme weather events affecting the external grid). The methodology is demonstrated in case studies focused on representative scenarios in Brazil, in which the value of load lost and the costs associated with repair of damaged grid components and investment in mitigation options are calculated and further compared. Ultimately, the analysis allows for the selection of the most appropriate strategy in terms of resilience and/or costs over a range of scenarios.

The text is organized as follows. Section II describes the materials and methods, Section III presents the case studies, Section IV contains results and discussions, and Section V presents the conclusions.

\section{MATERIALS AND METHODS}

This section describes extreme events affecting low-income neighborhoods (Section II-A) and presents the problem formulation (Section II-B), including impact assessment and mitigation strategies as follows.

\section{A. Problem description}

Electricity infrastructures are often in trouble due to natural hazards, such as floods and landslides, extreme weather conditions, such as heavy rain and windstorms, and human-made attacks, such as vandalism and theft of energy or electrical equipment. In low-income households and communities, the consequences of such extreme events are further amplified due to the network topology and irregularities combined with social poverty [13], as explained as follows.

Power distribution networks in low-income neighborhoods are typically radial, with households connected to the main distribution feeder at low voltage level every few meters. The line rating tends to be relatively low because of the low energy intensity of low-income households, but is usually higher than expected due to irregular users connected to the grid. As a consequence, power distribution networks in lowincome neighborhoods are less reliable than in high-income neighborhoods and prone to outages under extreme operating conditions.

The impact of an outage in low-income neighborhoods involves both the infrastructure and service and also depends on the financial means available to restore the out-of-service loads. Consequently, the supply interruption times may be very long or even permanent due to the impossibility of reconnecting to the grid, whereas the maintenance cost and the value of load lost may be much higher than the investment cost in mitigation strategies towards a resilient grid. This motivates the search for feasible solutions able to improve power system resilience of low-income neighborhoods to extreme events. Ultimately, it will guarantee that the energy needs of the whole society are met adequately and also help to keep low-income communities safe.

\section{B. Problem formulation}

The impact of extreme events on electricity infrastructures is reflected on damaged equipment and on the amount of energy not supplied. Extreme events may affect individual households in vulnerable areas, as well as distribution lines, poles, transformers, and other auxiliary equipment not protected. To this extent, the damages to individual grid components and to the physical infrastructure may be repairable or request full replacement, depending on the impact of the extreme event in consideration on the grid components (i.e., from minor to major). In turn, the interruption duration depends on the impact on the energy infrastructure and on the restoration time and may last for a few hours (for example, when caused by the removal of grid components) or forever in case of permanent disconnection from the grid (for example, when caused by non-replaceable destruction of property or loss of life). The amount of energy not supplied is equivalent to the expected energy demand not supplied over time. In case of permanent disconnection from the grid, the energy not supplied is infinite (worst-case scenario).

Thereby, the methodology computes the costs of repair or replacement of damaged infrastructures of each component $i=1, \cdots, N_{C}$ affected, denoted by $c_{r e, i}$, and the the value of loads lost of each load $i=1, \cdots, N_{L}$ affected, denoted by $c_{\text {out }, i}$, over the time horizon $\tau$. Next, the sum of these costs is compared with the cost of implementation of mitigation alternatives for each component $i=1, \cdots, N_{C}$ affected, denoted by $c_{i n s t, i}$. This comparison enables to determine if investing on resilience enhancements is more advantageous than doing nothing for impact mitigation. In this context, some particularities are described as follows.

Equation (1) describes $c_{r e, i}$ as a function of the damage level $d_{i} \in[0,1]$ over the time horizon $\tau$, considering a maximum cost $\overline{c_{r e, i}}$ over $\tau$ for each component $i=1, \cdots, N_{C}$.

$$
c_{r e, i}=d_{i} \times \overline{c_{r e, i}}, \text { for } i=1, \cdots, N_{C}
$$

The decision of implementing a specific mitigation strategy is formulated as a long-term cost minimization function over $\tau$, considering the costs associated with the installation of mitigation options, repair of damaged components, and amount of energy not supplied. For the utility company, the decision is justified if the implementation cost is lower than the maintenance and outage costs for not hardening individual components and structures, that is, if (2) holds for some $i=1, \cdots, N_{C}$.

$$
c_{\text {inst }, i} \leq c_{\text {out }, i}+c_{r e, i}
$$

In turn, for the community and household owners, the decision of installing off-grid alternative sources of energy for island-mode operation against extreme events is justified if the implementation cost is lower than the maintenance and outage costs, considering the costs that are not subsidized by the government. Thereby, (2) is turned into (3) in the case of low-income community and households, with $i=1, \cdots, N_{L}$ 
referred to loads and $s_{\text {inst }, i}, s_{\text {out }, i}, s_{\text {re }, i} \in[0,1]$ denoting the per unit costs that are not subsidized by the government with regards to implementation, energy consumption, and maintenance, respectively.

$$
s_{\text {inst }, i} c_{\text {inst }, i} \leq s_{\text {out }, i} c_{\text {out }, i}+s_{r e, i} c_{r e, i}
$$

As the occurrence of a natural disaster, extreme weather event, or human-made attack is often uncertain, a probabilistic modeling may be assumed to account for failure of individual grid components, as in [5]. This approach allows for a probabilistic evaluation of outages triggered by specific probabilistic conditions. In this situation, (2) and (3) become (4) and (5), where $\mathcal{P}(x)$ is the probability of the value $x$ of a specific variable of interest (e.g. wind speed, precipitation, electric current) to occur and lead to an outage. To this extent $\mathcal{P}(x)$ may be obtained from fragility curves of specific grid components, historical data, expectations, among others.

$$
\begin{gathered}
c_{\text {inst }, i} \leq \mathcal{P}(x)\left(c_{\text {out }, i}+c_{\text {re }, i}\right) \\
s_{\text {inst }, i} c_{\text {inst }, i} \leq \mathcal{P}(x)\left(s_{\text {out }, i} c_{\text {out }, i}+s_{\text {re }, i} c_{\text {re }, i}\right)
\end{gathered}
$$

\section{CASE STUDY}

In this section, two distinct scenarios are taken into consideration to evaluate whether the decision of improving grid resilience against extreme events is feasible in low-income neighborhoods, inspired in Brazil. Notably, the following mitigation strategies are taken into consideration: (1) deploying intelligent electronic devices to detect and eliminate thefts of energy (Section III-B), and (2) installing off-grid photovoltaic systems or independent generators at household or community level for autonomous operation from the external grid against supply interruption (Section III-C). These choices are made because the first scenario contemplates upgrades on the power distribution network for hardening infrastructure, whereas the second scenario includes distinct off-grid generators at household or community level to provide autonomous operation from the external grid.

\section{A. Premises}

The value of loads lost is equivalent to $c_{\text {out }, i}=c_{\text {out }, i}^{\prime} \times$ $e_{\text {out }, i}$, with unit cost per energy $c_{\text {out }, i}^{\prime}=0.75 R \$ / \mathrm{kWh}$ and energy consumption $e_{\text {out }, i}$ of an individual household between 30 and $220 \mathrm{kWh} /$ month [14]. Even though the government usually subsidizes the electricity bills of low-income households usually (partially or totally), the value of loads lost is still supposed to be paid in full for the distribution utility company.

Regarding electrical components, the unit costs $\left(c_{i n s t, i}^{\prime}\right.$, $\left.c_{r e, i}^{\prime}\right)$ in use for overhead power lines are equivalent to $80 R \$ / \mathrm{m}$, with the costs in the Brazilian Real currency $R \$$, and total costs $c_{i n s t, i}=c_{r e, i}=80 \times l_{i}$ in $\mathrm{R} \$$, with $l_{i}$ representing the line length in $\mathrm{m}$. The investment cost of an intelligent electronic device for smart metering is equal to
TABLE I

OFF-GRID PHOTOVOLTAIC SYSTEM COST PARAMETERS

\begin{tabular}{ll}
\hline \hline Power $(\mathrm{kWp})$ & $c_{\text {inst }, i}(\mathrm{R} \$)$ \\
\hline 1 & 2500 \\
2 & 5000 \\
5 & 7500 \\
6 & 10000 \\
10 & 12500 \\
\hline \hline
\end{tabular}

$100 R \$$ for a single-phase device and $300 R \$$ for a three-phase device, whereas their respective maintenance costs correspond to $20 R \$$ and $50 R \$$ per year. In turn, the installation costs of off-grid photovoltaic panels with batteries are given in Table I.

\section{B. Situation \#1}

In this situation, the feasibility of deploying intelligent electronic devices to monitor electric variables and detect energy thefts is evaluated in two settings: (1) along the distribution lines and (2) at the connection points of the households with the external grid through metered supply.

In this case, $c_{\text {out }, i}, i=1, \cdots, N_{L}$, corresponds to the value of loads that are not accounted due to irregular supply. In turn, the number of metering devices is denoted by $N_{I E D}$, with $c_{i n s t, i}$ and $c_{r e, i}$ given in Section III-B, $i=1, \cdots, N_{I E D}$. They are supposed to be three-phase devices along the distribution lines and single-phase at the connection points of the households with the external grid.

Considering that the implementation costs are paid in full by the utility company, (2) can be re-written as (6) as follows.

$$
\sum_{i=1}^{N_{I E D}} c_{i n s t, i}>\sum_{i=1}^{N_{L}} c_{\text {out }, i}+\sum_{i=1}^{N_{I E D}} c_{r e, i}
$$

\section{Situation \#2}

This section evaluates the installation of off-grid photovoltaic panels combined with batteries at household or community level for autonomous operation from the external grid against human-made attacks. In this case, the affected lines are located after the point of connection to the customers and belong to the household owners.

The installation costs given in Table I are considered to calculated the implementation costs associated with offgrid photovoltaic systems, whereas the cost associated with replacement of destroyed or stolen lines are given by the installation cost of a new overhead line, i.e., $c_{r e, i}=80 \times l_{i}$ $\mathrm{R} \$$.

In addition, the costs associated with the power outage are reflected in the operation costs associated, for example, with independent generators (e.g. diesel) and non-electrified appliances (e.g. wood ovens and candles). These costs are assumed to be at least three times more expensive than the electricity cost, i.e., $c_{\text {out }, i}^{\prime}=2.25 R \$ / \mathrm{kWh}$, whereas the emergency energy consumption is cut to half over the outage duration (that is, ranging from 15 to $110 \mathrm{kWh} / \mathrm{month}$ ). 
TABLE II

RESULTS: $t_{\text {out }}$ (MONTHS) IN \#1

\begin{tabular}{lllll}
\hline \hline Setting & \multicolumn{2}{l}{ Household } & \multicolumn{2}{l}{ Distribution } \\
\hline$e_{\text {out }, i}(\mathrm{kWh} / \mathrm{mon}$ th $)$ & 30 & 220 & 30 & 220 \\
\hline$N_{L}=1$ & 12.5 & 1.8 & 11.25 & 1.8 \\
$N_{L}=3$ & 4.15 & 0.6 & 4.2 & 0.6 \\
$N_{L}=5$ & 2.5 & 0.36 & 2.6 & 0.4 \\
\hline \hline
\end{tabular}

As $c_{r e, i}$ is a function of the line length, the total line length replaced can be written as a function of the frequency of occurrence, denoted by $f_{d}$, over the time horizon $\tau$, that is, $l_{i}=l_{i}^{\prime} f_{d} \tau$. Thereby, the time horizon when the repair and outage costs compensate for the cost of implementation of the mitigation option, given by (7), can be obtained from (5), with $\mathcal{P}(x)=f_{d}$.

$$
\tau \geq \frac{s_{\text {inst }, i} c_{\text {inst }, i}}{\left(c_{\text {out }, i}^{\prime} \times e_{\text {out }, i}+c_{r e, i}^{\prime} \times l_{i}^{\prime}\right) f_{d}}
$$

\section{RESUlTS}

In this section, the scenarios described in Sections III-B and III-C are evaluated in distinct settings to determine whether the decision of improving grid resilience against extreme events is feasible in low-income neighborhoods.

\section{A. Situation \#1}

Consider a distribution feeder with $N_{L}$ irregular customers every $5 \mathrm{~m}$ and the following settings: $N_{I E D}=1$ (1) along the distribution lines every $5 \mathrm{~m}$ and (2) at the connection point of the households with the external grid. In both settings, $c_{\text {out }, i}, i=1, \cdots, N_{L}$ may vary from $0.75 \times 30 \times 12 \times t_{\text {out }}$ to $0.75 \times 220 \times 12 \times t_{\text {out }} \mathrm{R} \$$ per household, where $t_{\text {out }}$ is the duration of the irregular supply in years. Hence, $c_{r e, i}=$ $50 \times t_{\text {out }} \mathrm{R} \$$ in the first setting and $c_{r e, i}=20 \times t_{\text {out }} \mathrm{R} \$$ in the second setting, $i=1, \cdots, N_{I E D}$. Finally, the outage duration $t_{\text {out }}$ that compensates for the mitigation alternatives aforementioned can be calculated as a function of the costs. The results are displayed in Table II according to $N_{L}, e_{o u t, i}$, and the installation setting in consideration.

If $t_{\text {out }}$ is shorter than the lifespan of the metering infrastructure, then its implementation is recommended. Assuming that it remains in operation for at least 36 months for the purpose of this analysis, then its implementation is justified in all settings of Table II.

\section{B. Situation \#2}

Assume $l_{i}^{\prime}=2 \mathrm{~m}$ for a single household, an outage duration $t_{\text {out }}=48 \mathrm{~h}$ per occurrence, and the emergency energy consumption $e_{\text {out }, i}$ equivalent to half of the power supplied by each corresponding off-grid system during $t_{\text {out }}$. Considering $s_{\text {inst }, i}=1$ (no installation subsidized) and $s_{\text {inst }, i}=0.5(50 \%$ subsidized), with $s_{r e, i}=1$ (that is, no replacement subsidized) and $s_{\text {out }, i}=0$ in normal operation for simplification (that is, energy consumption $100 \%$ subsidized in normal operation), the results of (7) are summarized in Tables III and IV as a
TABLE III

RESULTS: $\tau$ (YEARS) IN \#2 WITHOUT SUBSIDIZATION

\begin{tabular}{llll}
\hline \hline Power $(\mathrm{kWp})$ & \multicolumn{4}{l}{$\tau$ (years) } \\
\hline$f_{d}\left(\right.$ year $\left.^{-1}\right)$ & 1 & 2 & 3 \\
\hline 1 & 15.4 & 7.2 & 5.2 \\
2 & 30.4 & 15.2 & 10.2 \\
5 & 44.6 & 22.3 & 14.9 \\
6 & 58.7 & 29.4 & 19.6 \\
10 & 70.8 & 35.4 & 23.6 \\
\hline \hline
\end{tabular}

TABLE IV

RESULTS: $\tau$ (YEARS) IN \#2 WITH $s_{\text {inst }, i}=0.5$

\begin{tabular}{llll}
\hline \hline Power $(\mathrm{kWp})$ & \multicolumn{2}{l}{$\tau$ (years) } \\
\hline$f_{d}\left(\right.$ year $\left.^{-1}\right)$ & 1 & 2 & 3 \\
\hline 1 & 7.7 & 3.6 & 2.6 \\
2 & 15.2 & 7.6 & 5.1 \\
5 & 22.3 & 11.2 & 7.5 \\
6 & 29.4 & 14.7 & 9.8 \\
10 & 35.4 & 17.7 & 11.8 \\
\hline \hline
\end{tabular}

function of $f_{d}$, with $f_{d}=1,2,3$ per year, and the peak power provided by each off-grid system.

\section{Discussion}

The feasibility of enhancing grid resilience in low-income neighborhoods can be interpreted in different ways according to the extreme event of interest. To this extent, the probability of occurrence, impact, and possible mitigation alternatives make an impact on the decision process.

In the first situation, the results are influenced by the implementation costs and energy consumption form irregular customers. In some cases, the return of investment is almost immediate, while in others it may take longer for this to happen. Overall, the return of investment is expected to occur for all settings in less than 2 months when $e_{\text {out }, i}=$ $220 \mathrm{kWh} /$ month and in roughly a year when $e_{\text {out }, i}=30$ $\mathrm{kWh} /$ month.

In the second situation, the results are also influenced by the implementation costs not subsidized and the expected frequency of occurrence. In this case, however, the return of investment is more likely to occur when the energy consumption is lower, as the implementation cost of the corresponding offgrid photovoltaic system is cheaper in these cases. Nonetheless, the results show that the subsidization provided by the government may lead to a faster return of investment.

Overall, the results suggest that the total costs associated with maintenance and energy consumption may compensate for the investment costs associated with alternatives for enhancing grid resilience in low-income neighborhoods. Nevertheless, the results indicate that upgrades in the electricity network infrastructure are more cost-effective than islandmode operation, as the return of the investment is significantly shorter in all simulated settings.

The results previously obtained were calculated with a deterministic analysis (that is, using constant demand profiles 
and frequencies of occurrence over time) that does not consider the temporal value of money. Despite that, the methodology is general enough to handle non-deterministic values and timevarying costs. In this case, the constant demand profiles, event frequencies, and costs involved in the analysis must be replaced with varying ones.

\section{CONCLUSION}

This article has evaluated the costs and benefits concerning power system resilience enhancements in low-income neighborhoods, considering distinct events of interest that may lead to power outages. The decision criteria compares the cost associated with implementation of mitigation alternatives with the value of load lost and the cost to repair damaged components to determine the benefits of different strategies for power system resilience improvements targeted at lowincome neighborhoods. The results of different case studies indicate that the maintenance costs plus the value of load lost may compensate for the investment costs associated with resilience improvements in a long-term horizon, as the return of investment may be relatively long in some cases.

\section{REFERENCES}

[1] N. Scott, K. McKemey, and S. Batchelor, "Energy in low-income urban communities," February 2005.

[2] G. C. A. Nadaud, "Acesso à Energia Elétrica de Populaćões Urbanas de Baixa Renda: O Caso das Favelas do Rio de Janeiro," Master's thesis, Universidade Federal do Rio de Janeiro, Brazil, 2012.

[3] "Energy Equity: Bringing Solar Power to Low-Income Communities," https://e360.yale.edu/features/energy-equity-bringing-solar-powerto-low-income-communities, accessed: 2020-05-11.

[4] M. Panteli, D. N. Trakas, P. Mancarella, and N. D. Hatziargyriou, "Power systems resilience assessment: Hardening and smart operational enhancement strategies," Proceedings of the IEEE, vol. 105, no. 7, pp. 1202-1213, July 2017.

[5] M. Panteli, C. Pickering, S. Wilkinson, R. Dawson, and P. Mancarella, "Power system resilience to extreme weather: Fragility modeling, probabilistic impact assessment, and adaptation measures," IEEE Transactions on Power Systems, vol. 32, no. 5, pp. 3747-3757, Sep. 2017.

[6] T. Lagos, R. Moreno, A. N. Espinosa, M. Panteli, R. Sacaan, F. Ordonez, H. Rudnick, and P. Mancarella, "Identifying optimal portfolios of resilient network investments against natural hazards, with applications to earthquakes," IEEE Transactions on Power Systems, vol. 35, no. 2, pp. 1411-1421, March 2020.

[7] K. Anderson, X. Li, S. Dalvi, S. Ericson, C. Barrows, C. Murphy, and E. Hotchkiss, "Integrating the value of electricity resilience in energy planning and operations decisions," IEEE Systems Journal, pp. 1-11, 2020.

[8] G. Li, G. Huang, Z. Bie, Y. Lin, and Y. Huang, "Component importance assessment of power systems for improving resilience under wind storms," Journal of Modern Power Systems and Clean Energy, vol. 7, no. 4, pp. 676-687, July 2019 .

[9] S. Espinoza, A. Poulos, H. Rudnick, J. C. de la Llera, M. Panteli, and P. Mancarella, "Risk and resilience assessment with component criticality ranking of electric power systems subject to earthquakes," IEEE Systems Journal, pp. 1-12, 2020.

[10] A. Bagheri, C. Zhao, F. Qiu, and J. Wang, "Resilient transmission hardening planning in a high renewable penetration era," IEEE Transactions on Power Systems, vol. 34, no. 2, pp. 873-882, March 2019.

[11] A. Hussain, S. D. A. Shah, and S. M. Arif, "Heuristic optimisationbased sizing and siting of dgs for enhancing resiliency of autonomous microgrid networks," IET Smart Grid, vol. 2, no. 2, pp. 269-282, 2019.

[12] E. B. Watson and A. H. Etemadi, "Modeling electrical grid resilience under hurricane wind conditions with increased solar and wind power generation," IEEE Transactions on Power Systems, vol. 35, no. 2, pp. 929-937, March 2020.
[13] Y. Andersson-Sköld and L. Nyberg, "Effective and sustainable flood and landslide risk reduction measures: An investigation of two assessment frameworks," International Journal of Disaster Risk Science, vol. 7, no. 4, pp. 374-392, Dec 2016.

[14] "Tarifa Social de Energia $\quad$ Elétrica," https://www.eneldistribuicaosp.com.br/para-sua-casa/tarifa-socialde-energia-eletrica, accessed: 2020-05-08. 